\title{
The Doppler Spectra of Medium Grazing Angle Sea Clutter; Part 2: Model Assessment and Simulation
}

\author{
Simon Watts ${ }^{1,4}$, Luke Rosenberg ${ }^{2,3}$, Stephen Bocquet ${ }^{2}$, Matthew Ritchie ${ }^{4}$ \\ ${ }^{1}$ Consultant, United Kingdom \\ ${ }^{2}$ Defence Science and Technology Group, Australia \\ ${ }^{3}$ University of Adelaide, Australia \\ ${ }^{4}$ UCL, London, United Kingdom \\ Email: simon.watts.gb@ieee.org
}

\begin{abstract}
This paper is concerned with the assessment of models of Doppler spectra, derived from high range resolution X-band radar sea clutter observed from an airborne platform over the range of grazing angles, $15^{\circ}$ to $45^{\circ}$. When looking up or downwind these models represent the strong correlation between mean Doppler shift and local spectrum intensity. When combined with random fluctuations of spectrum width, these characteristics give the spectra a temporal and spatial variability. The models are used to predict clutter spectrum statistics as a function of Doppler frequency and these are compared with statistics derived from the original data. It is also shown how realistic range-varying coherent clutter returns can be simulated using the models.
\end{abstract}

Keywords — Radar sea clutter, Doppler spectra, modelling and simulation, clutter statistics

\section{Introduction}

In Part 1 of this paper [1] coherent data from the Ingara radar [2] was analysed to assess the characteristics of the Doppler spectra of the returns. Ingara is a fully-polarimetric X-band radar system maintained and operated within the Defence Science and Technology (DST) Group ${ }^{1}$ in Australia [2]. The dataset comprises two trials on separate occasions and in two distinctly different regions. The first sea-clutter trial was conducted in 2004 in the Southern Ocean approximately $100 \mathrm{~km}$ south of Port Lincoln, South Australia. The site chosen was at the edge of the South Australian continental shelf where there was little chance of shallow water affecting the wave field. During the trial, ocean backscatter was collected for a range of different geometries on eight separate days with different ocean conditions. The second trial in 2006 was conducted in littoral and open ocean environments near Darwin in the Northern Territory. It was shown in [1] that the modelling approach originally described in [3,4] for lower grazing angle data was likely to be a good fit to the medium grazing angle data from Ingara. The Doppler spectra of sea clutter at medium grazing angles can be expected to be highly variable, both spatially and temporally, for a wide range of

\footnotetext{
${ }^{1}$ Formerly Defence Science and Technology Organisation (DSTO)
} 
environmental conditions and radar polarisations. This variability can be described in terms of two main characteristics, as observed in $[3,4]$ and shown in the results presented in [5,6] and in this paper. Firstly, the width of the power spectra, expressed as the standard deviation of the Doppler frequencies, fluctuates over range and time in a manner that is mainly independent of the local spectrum intensity. Secondly, the mean Doppler shifts of the local spectra show a distinct correlation with local spectrum intensity. This is particularly evident when looking up or downwind, but is also observed to varying degrees for most look directions. This variation of mean Doppler shift for low grazing angle clutter has been reported by various researchers over many years [7-10]. In [3,4], a model was developed to capture this behaviour so that it could be applied to the modelling and simulation of sea clutter Doppler spectra at low grazing angles. In this paper, it is shown how models for Doppler spectra based on observations at medium grazing angles have been developed and how they can be exploited in the design and development of radar systems. Some initial results of this work were presented in [5]. A summary of the model, described in detail in [1], is given in Section 2, defining the various parameters of the model. An important aspect of the modelling method is to be able to predict the values of the parameters of the models under different viewing geometries, radar polarisations and environmental conditions. In Section 3, a general model is described and then used to capture the parameters associated with the bimodal behaviour of Doppler spectra.

When the local intensity is modelled with a suitable probability density function (PDF), such as the compound K distribution, with added thermal noise, it is possible to predict the typical amplitude statistics in individual Doppler bins, as observed over a period of time or over a range interval. In Section 4, the performance or accuracy of these models are then analytically assessed. With the addition of suitable models of temporal or spatial correlation of the intensity, the Doppler spectrum models can be exploited to simulate realistic Doppler spectra. Some work on the simulation of Doppler spectra had been previously reported in [11] and other potential methods have also previously been proposed, such as [12]. Those methods can go some way towards reproducing examples of Doppler spectra but do not allow the generation of continuous coherent returns from which realistic spectra can be derived. The generation of realistic range-varying coherent data is described in Section 5. Finally, in Section 6 there is a discussion of the comparison between these results and those previously developed for low grazing angle clutter.

\section{Summary of the model}

In [1], a general model for the power spectrum of the clutter alone (i.e. without the added thermal noise) is given by a mixture model of two Gaussian-shaped power spectra:

$$
\begin{aligned}
\mathbf{G}\left(f, x_{n}, s_{1}, s_{2}\right)= & \frac{\alpha x_{n}}{\sqrt{2 \pi} s_{1}} \exp \left[-\frac{\left(f-m_{f 1}\left(x_{n}\right)\right)^{2}}{2 s_{1}^{2}}\right] \\
& +\frac{\beta x_{n}}{\sqrt{2 \pi} s_{2}} \exp \left[-\frac{\left(f-m_{f 2}\left(x_{n}\right)\right)^{2}}{2 s_{2}^{2}}\right]
\end{aligned}
$$


where $\alpha+\beta=1(\alpha, \beta>0), x_{n}$ is the local clutter intensity with range index $n$ and $s_{1}$ and $s_{2}$ are the standard deviations of the spectrum components. The mean Doppler shifts of the two spectrum components are modelled by

$$
\begin{aligned}
m_{f 1}\left(x_{n}\right) & =A+B x_{n}+r, \quad x_{n} \leq t \\
& =A+B t+r, \quad x_{n}>t \\
m_{f 2}\left(x_{n}\right) & =A+B x_{n}+r .
\end{aligned}
$$

The parameters $A$ and $B$, for a given set of environmental conditions and radar parameters, represent the intercept and slope of a linear fit to the variation of measured values of $m_{f}\left(x_{n}\right)$ as a function of $x_{n}$ for those spectra with $x_{n}$ less than the threshold $t$. This threshold is defined as the intensity above which the rate of variation of $m_{f}\left(x_{n}\right)$ with $x_{n}$ changes (see [1]). The parameters $A, B$ and $t$ will have values that are dependent on the environmental conditions and radar parameters, as discussed later. The dependence of mean Doppler shift on the local intensity for $x_{n}>t$, as modelled in (2), has the effect of broadening the spectrum if $\alpha \neq 0$ (or $\beta \neq 1$ ) and $x_{n}$ exceeds some threshold, $t$, providing a representation of the bimodal behaviour seen in some data, as illustrated in [1]. The use of a threshold is suggested by the distinct change above some intensity level in the dependence of $m_{f}\left(x_{n}\right)$ on $x_{n}$ seen in some data sets. The parameter $r$ is the difference between the actual value $m_{f}\left(x_{n}\right)$ and the value represented by a fit based on $A, B$ and $t$ in (2). This parameter is modelled as a Gaussian random variable with zero mean and standard deviation, $\sigma_{r}$, having a PDF, $p_{r}(r)$. In the results presented here, the model is developed using a parameter $s=s_{1}=s_{2}$. This assumption was found to be appropriate for our data, but clearly other fits to the model could be attempted with $s_{1} \neq s_{2}$, if evidence for that were found. However, as discussed in [1], the normalised spectra were observed to be a good fit to a Gaussian shape at lower values of intensity (i.e. $x_{n} \leq t$ ) and the combined spectra modelled using (1) would no longer have an average Gaussian shape for $x_{n} \leq t$ if $s_{1} \neq s_{2}$

The standard deviation of the spectrum width is modelled as being a random variable, $s$, which is independent of $x_{n}$. In [4], the $\operatorname{PDF}$ of $s, p_{s}(s)$, is modelled as having a normal distribution, with mean $m_{s}$ and variance $\sigma_{s}^{2}$. The values of $s$ must of course be restricted to $s>0$. In practice it is found that $m_{s} \gg \sigma_{s}$ and so modelling $s$ in this way does not cause any problems. Now

$$
p_{s}(s)=\frac{1}{\sqrt{2 \pi} \sigma_{s}} \exp \left(\frac{-\left(s-m_{s}\right)^{2}}{2 \sigma_{s}^{2}}\right) ; s>0
$$

Alternatively, the spectrum width can be modelled as having a gamma distribution, which is also a good fit to the data. Now

$$
p_{s}(s)=\frac{1}{\Gamma(\mu) c^{\mu}} s^{\mu-1} \exp \left(-\frac{s}{c}\right)
$$

where the shape, $\mu=m_{s}^{2} / \sigma_{s}^{2}$ and scale, $c=\sigma_{s}^{2} / m_{s}$. 


\section{General modelling of parameters}

In order to characterise the bi-modal modelling scheme presented in this paper, there are a large number of input parameters required. In previous publications $[4,5,13]$ these have been extracted directly from example data. However, Rosenberg et al. [6] has recently presented an empirical model which relates these input parameters to the polarisation, collection geometry and sea-state. This model was influenced by the work of Crisp et al. [14] who proposed a similar model specifically for the $\mathrm{K}$ distribution shape parameter. The new version is a variation designed to better model other parameters of interest which have a stronger component due to the swell. This section provides a brief summary of this model with further details given in [6]. In this recent publication, the new model was applied to both the K and Pareto distribution shape parameters, the two components of the linear frequency centroid model, the mean and standard deviation of the spectral width Gaussian model and the spatial correlation. For the work in this paper, the model has now been applied to capture the spread of the fluctuations around the mean Doppler, $\sigma_{r}$ and the four bimodal model components. These include the y-intercept, $A$, the initial slope, $B$, the threshold, $t$ and the ratio, $\beta$ between the two components. Section 3.1 describes further details about how these parameters were adapted to the parameter model.

The model here is defined in terms of a generic parameter $X$, defined for an azimuth look direction, $\phi$, and a grazing angle, $\theta$. Now the dependence of this generic parameter on wind and swell directions is modelled as the weighted sum of two components which represent the wind and swell directions. Assuming the data has been rotated so the upwind azimuth is now at $\phi=0^{\circ}$,

$$
X(\theta, \phi)=a_{0} \theta^{\gamma}\left[1+a_{1} \cos \phi+a_{2} \cos (2 \phi)+a_{3} \cos (\phi-\psi)+a_{4} \cos (2(\phi-\psi)]\right.
$$

where $\psi$ is the swell direction relative to upwind and $\gamma, a_{0}, \ldots a_{4}$ are the model coefficients. Representing the ocean conditions is difficult and the new model uses only the wind speed, $U$ and the significant wave height $H_{l / 3}$,

$$
Y=b_{0}+b_{1} \log _{10}(U)+b_{2} H_{1 / 3}
$$

where $b_{0}, b_{1}, b_{2}$ are the model coefficients. To relate these two models, the coefficients in (5) must be altered so they are independent of grazing angle. This is achieved by introducing a normalisation factor, $\theta_{0}$ and then redefining (5)

as

$$
X(\theta, \phi)=\left(\frac{\theta}{\theta_{0}}\right)^{\gamma}\left[\alpha_{0}+\alpha_{1} \cos \phi+\alpha_{2} \cos (2 \phi)+\alpha_{3} \cos (\phi-\psi)+\alpha_{4} \cos (2(\phi-\psi)]\right.
$$

where the new coefficients are related by

$$
\alpha_{0}=a_{0} \theta_{0}^{\gamma}, \alpha_{1}=a_{0} a_{1} \theta_{0}^{\gamma}, \ldots, \alpha_{4}=a_{0} a_{4} \theta_{0}^{\gamma}
$$


Implementing the model involves matching $\gamma$ and each of the $\alpha$ coefficients in (7). This in turn requires 6 sets of the $b$ parameters or 18 coefficients in total for each polarisation. Once the new coefficients in (8) are evaluated, they can be substituted into (7) with the normalising grazing angle $\theta_{0}$ set to $30^{\circ}$ to derive the final model value. It should be noted that this model is wholly empirical and has no known physical basis, but has been found to be able to represent the various parameters of interest here over a wide range of conditions.

\subsection{Modelling of bi-modal spectra}

Similar to the work in [6], modelling the parameters of interest requires estimates over a wide range environmental conditions and azimuth and grazing angles. To estimate the Doppler parameter models, a CPI of 64 pulses has been chosen, giving a $0.2 \mathrm{~s}$ observation time. This is repeated for each $5^{\circ}$ in azimuth and $2^{\circ}$ in grazing in order to limit the variation of the clutter-to-noise ratio (CNR) across the swath. Twelve days of data have been included covering seastates between 2 and 6 . This data comes from a fully-polarimetric collection with a PRF reduced to approximately $300 \mathrm{~Hz}$. Also due to the problems of estimating spectrum parameters in low CNR, only data regions with a CNR > 3 $\mathrm{dB}$ have been included (see [1], Section 2.4).

For a given set of conditions, the parameter $m_{f}\left(x_{n}\right)$ is estimated from the data for each power spectrum using the methods described in [1]. The parameters $A$ and $B$ are then estimated from linear fits to the variation of $m_{f}\left(x_{n}\right)$ with $x_{n}$ for those spectra with $x_{n}$ less than the threshold $t$. When modelling these parameters the intensity $x_{n}$ is normalised to have a mean of unity. Once $A, B$ and $t$ have been estimated, the parameters $\beta$ and $r$ can be determined. The parameter $r$ and is modelled as a Gaussian random variable with zero mean and standard deviation, $\sigma_{r}$. The parameter $\beta$ (see (1)) is determined by matching the observed rate of variation of $m_{f}\left(x_{n}\right)$ for $x_{n}>t$, with that predicted by applying (2) (see discussion in [1]). Finally, the parameter $s$ is derived for each spectrum as described in [1] and then $m_{s}$ and $\sigma_{s}$ are estimated by fitting a normal distribution to the measured mean and standard deviations of all range cells in the data block. Further discussion of the methods used to estimate parameter values can be found in Part 1 of this paper [1] and in [6].

To further improve the parameter modelling, it is useful to identify those fits where a linear model is sufficient to describe the spectrum (i.e. bimodal behaviour is not observed and $m_{f}\left(x_{n}\right)=A+B x_{n}+r$ for all values of $x_{n}$, or $\beta=1$ in (1)). For this purpose, the following criteria can be used:

- A threshold level at the minimum or maximum of the normalised intensity.

- A root mean square difference between the linear and bi-modal centre frequency fits of less than $2 \mathrm{~Hz}$.

- A ratio of components, $\beta \approx 1$. 
It was found that fits of the bimodal model to the data often returned extreme values of either $\beta \approx 0$ or $\beta \approx 1$. Therefore to improve the bi-modal parameter modelling, all apparent bi-modal fits which pass the criteria for linear behaviour given above have been modified to have $\beta=1$ (i.e. a linear fit) and an undefined threshold so they do not influence the bi-modal parameter model fit. To ensure that essentially linear behaviour is appropriately captured by the model, the modelled $\beta$ is further modified so any values that are above a defined threshold are also set to 1 . A threshold of 0.8 was determined by carefully comparing with the original data and minimising the fitting error. The sets of model coefficients for the mean Doppler spread, $\sigma_{r}$, the spatial de-correlation length, $R$ (described below), the four bi-modal parameters, $A, B, t$ and $\beta$ and the mean and standard deviation of the spectrum width, $m_{s}$ and $\sigma_{s}$, are shown in Tables A.1-A.4 in the appendix. These were derived using all the available Ingara data. The coefficients of the K and Pareto shape parameters were given in [6].

To demonstrate the parameter modelling, the F35 flight has been used with the model applied independently to each polarisation and compared with the original data. This flight has a sea-state of $4-5$ with a swell direction of $135^{\circ}$ relative to upwind. The wind speed was $10.2 \mathrm{~m} / \mathrm{s}$ and the wave height was $1.21 \mathrm{~m}$ which is relatively low given the wind speed. Examples of the fits of various parameters are shown in Figs. 1 to 5, where the azimuth angles are rotated so the upwind direction is centred at $0^{\circ}$, the downwind direction at $180^{\circ}$ and the cross wind directions at $90^{\circ}$ and $270^{\circ}$. Regions with missing or poor data are shown with diagonal lines/shading. In general, the model captures the behaviour of $\mathrm{HH}$ and $\mathrm{HV}$ polarised data quite well. The fit to VV data is not as good for some parameters, but is still able to reproduce much of the observed behaviour. It may also be noted that the VV data was in general much less spiky (see [6] and Table 1, below) and exhibited smaller variations of values of the spectrum model parameters than the other polarisations. The y-intercept and slope parameters, $A$ and $B$ are shown in Figs. 1 and 2. For the HH and HV polarisations, all 12 days have a periodicity across azimuth with a peak at upwind and small variation across grazing, confirming that the scattering from these channels are primarily wind driven. The results for the VV polarisation are more complicated with the slope peaking at downwind and also in regions corresponding to the swell or upwind directions. The average normalised root mean square errors for the three polarisations are $33.2 \%, 22.6 \%$ and $23.8 \%$ for the y-intercept, $A$ and $17.2 \%, 25 \%$ and $15.1 \%$ for the slope $B$.

Figures 3-5 then show example fits for the mean Doppler spread $\sigma_{r}$, the threshold $t$ and the component ratio, $\beta$. While the results for the threshold, $t$, and ratio, $\beta$, are quite noisy, the main trends have been captured by the model. Some of the modelled values are also slightly lower than the data due to the low wave height used for the modelling which has been balanced over the 12 days. Consequently, the average normalised root mean square errors for the three polarisations are $12.4 \%, 13.5 \%$ and $13.9 \%$ for the spread about the mean Doppler, $\sigma_{r}, 18.5 \%, 17.2 \%$ and $17.2 \%$ for 
the threshold, $t$, and $47.1 \%, 44.4 \%$ and $32.9 \%$ for $\beta$. Observed trends include a slightly higher values for $\sigma_{r}$ and $t$ for the $\mathrm{HH}$ and $\mathrm{HV}$ grazing regions above $30^{\circ}$. There is also a clear increase in $\sigma_{r}$ and $t$ for the $\mathrm{HH}$ polarisation in the swell direction. For the VV polarisation, there are clear peaks for $t$ along the crosswind directions, which also correspond to lower values of $\beta$, thus indicating bi-modal regions. For the HH polarisation, high $\beta$ values which correspond to linear fits are shown in the crosswind direction, while for $\mathrm{HV}$, the high $\beta$ values are in the upwind direction. To demonstrate the variation of model parameters, Table 1 provides a complete set of parameter models which form the basis of the simulation examples presented in Section 5. Two extra additions include the spatial decorrelation length, $\mathrm{R}$, and the time domain CNR. The decorrelation length is a measurement of when the spatial autocorrelation coefficient decays to $1 / \mathrm{e}$, while the CNR is modelled using the radar range equation with parameters defined in Table 2 and the IRSG-lin model for the mean backscatter [15,16]. Note that no multi-path or atmospheric effects have been included in this model. The impact of the platform motion can be included by adjusting the spectral width, $\sigma_{f}$, as discussed in [1]. With these example parameters, the additional spectrum spreading due to platform motion is calculated as $\sigma_{\text {antenna }}=49.64 \mathrm{~Hz}$. The sea state, $S$, in Table 2 was used to derive wind speed, $U$, and significant wave height, $H_{1 / 3}$, using the relationships [17] $U=3.2 S^{0.8}$ and $H_{1 / 3}=0.024 U^{2}$.
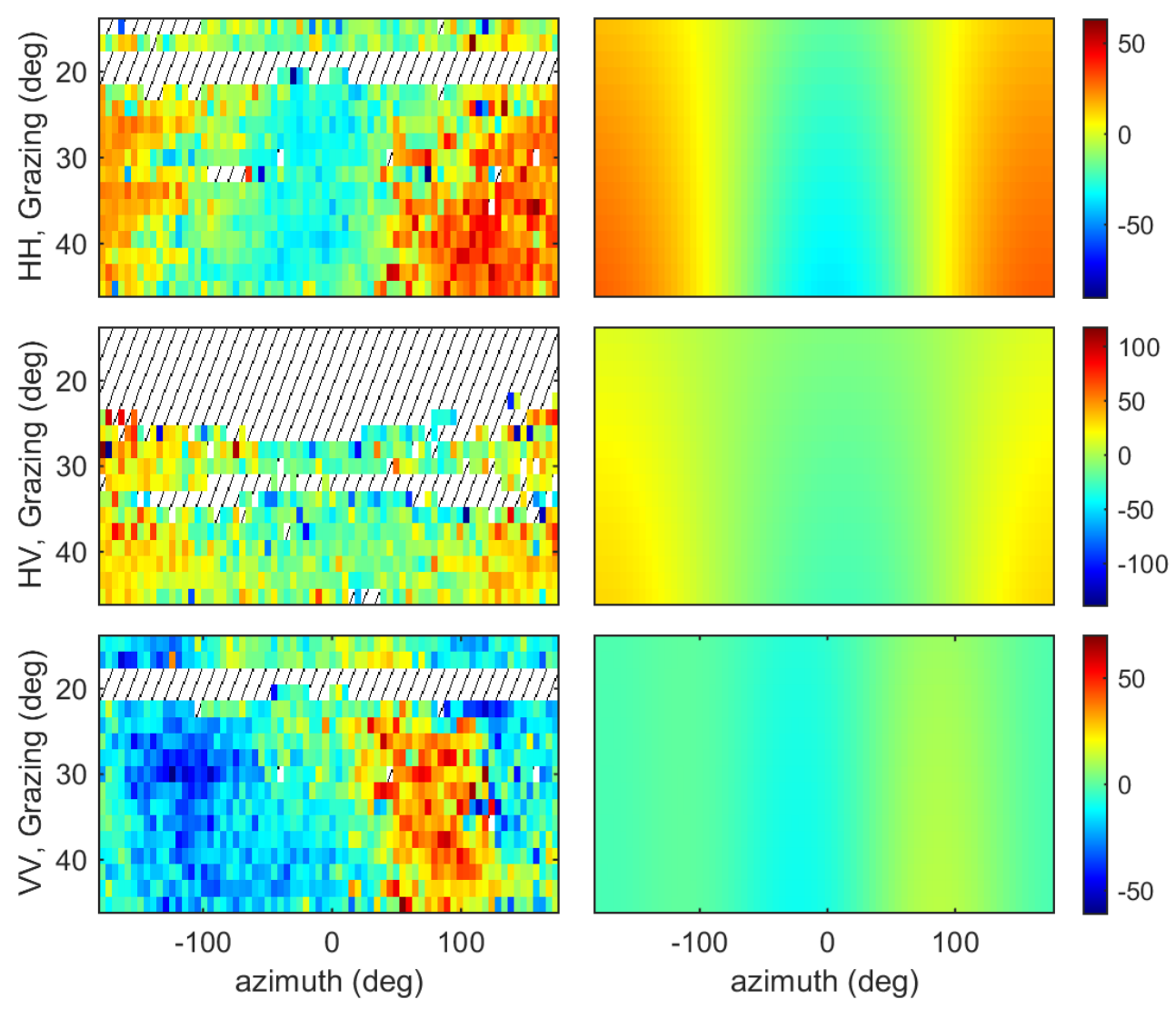

Figure 1 Variation of $y$-intercept, $A$ with azimuth and grazing. Data on left, parameter model on right. 

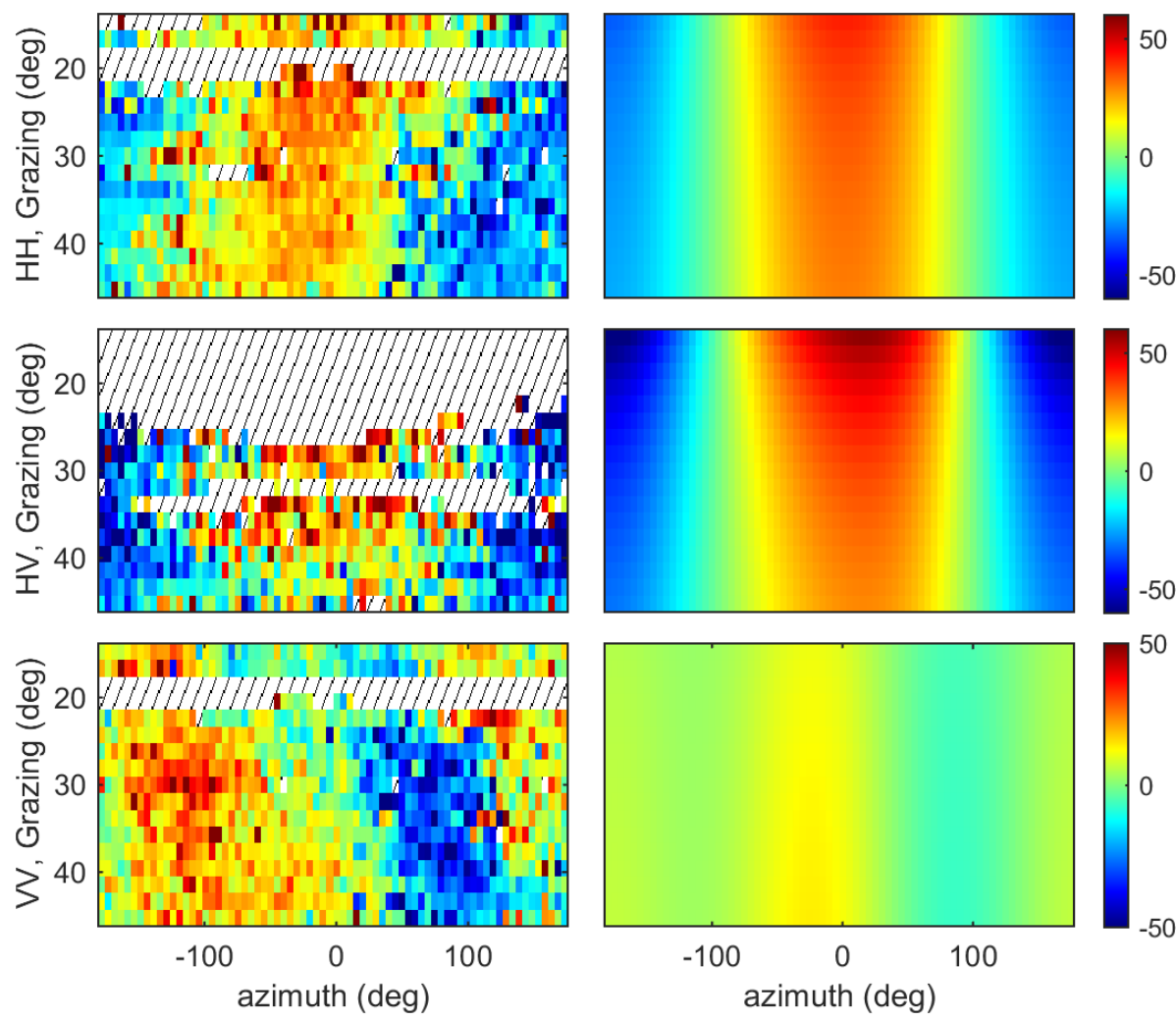

Figure 2 Variation of slope, $B$ with azimuth and grazing. Data on left, parameter model on right.
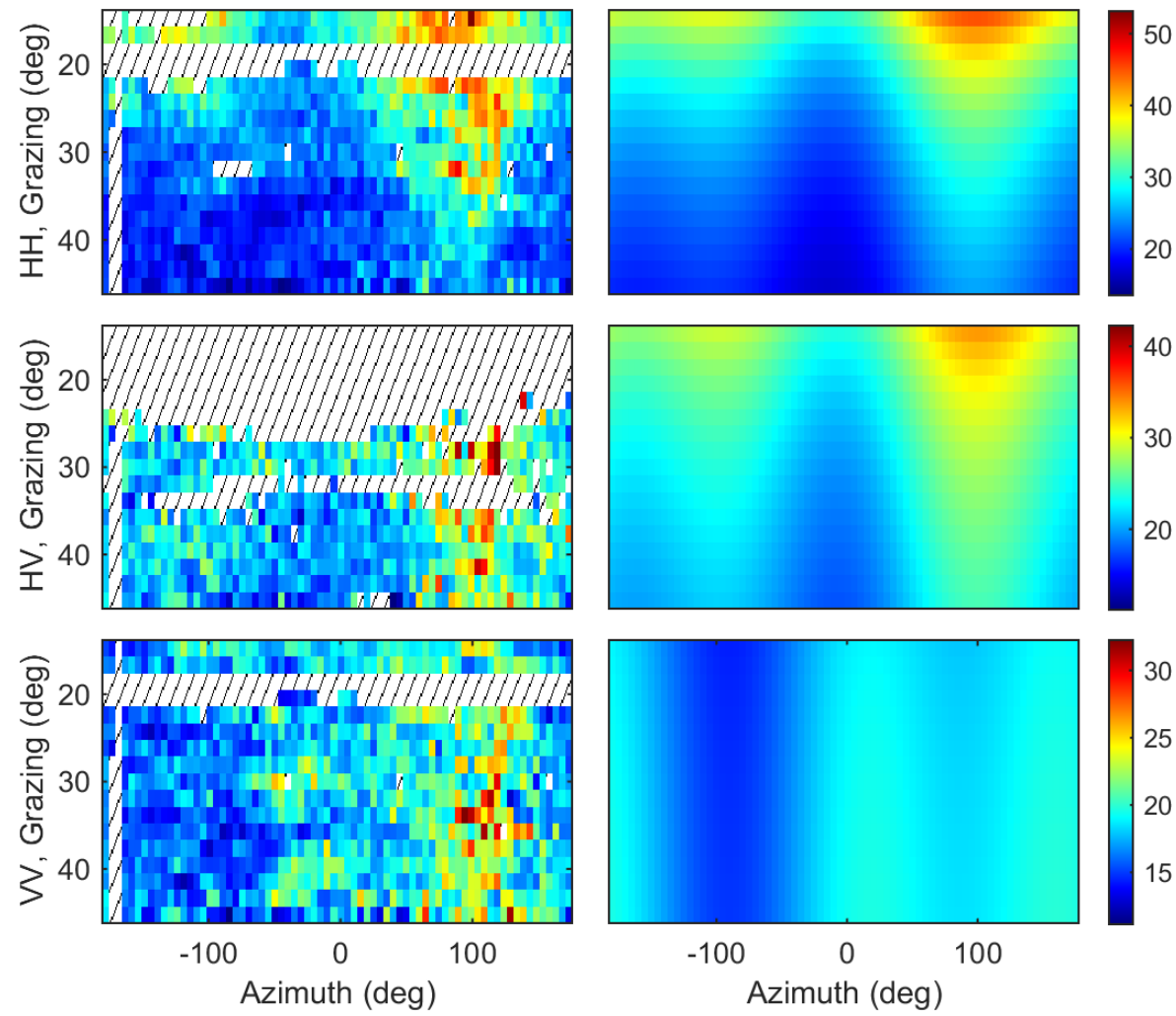

Figure 3 Variation of mean Doppler spread, $\sigma_{r}$ with azimuth and grazing. Data on left, parameter model on right. 

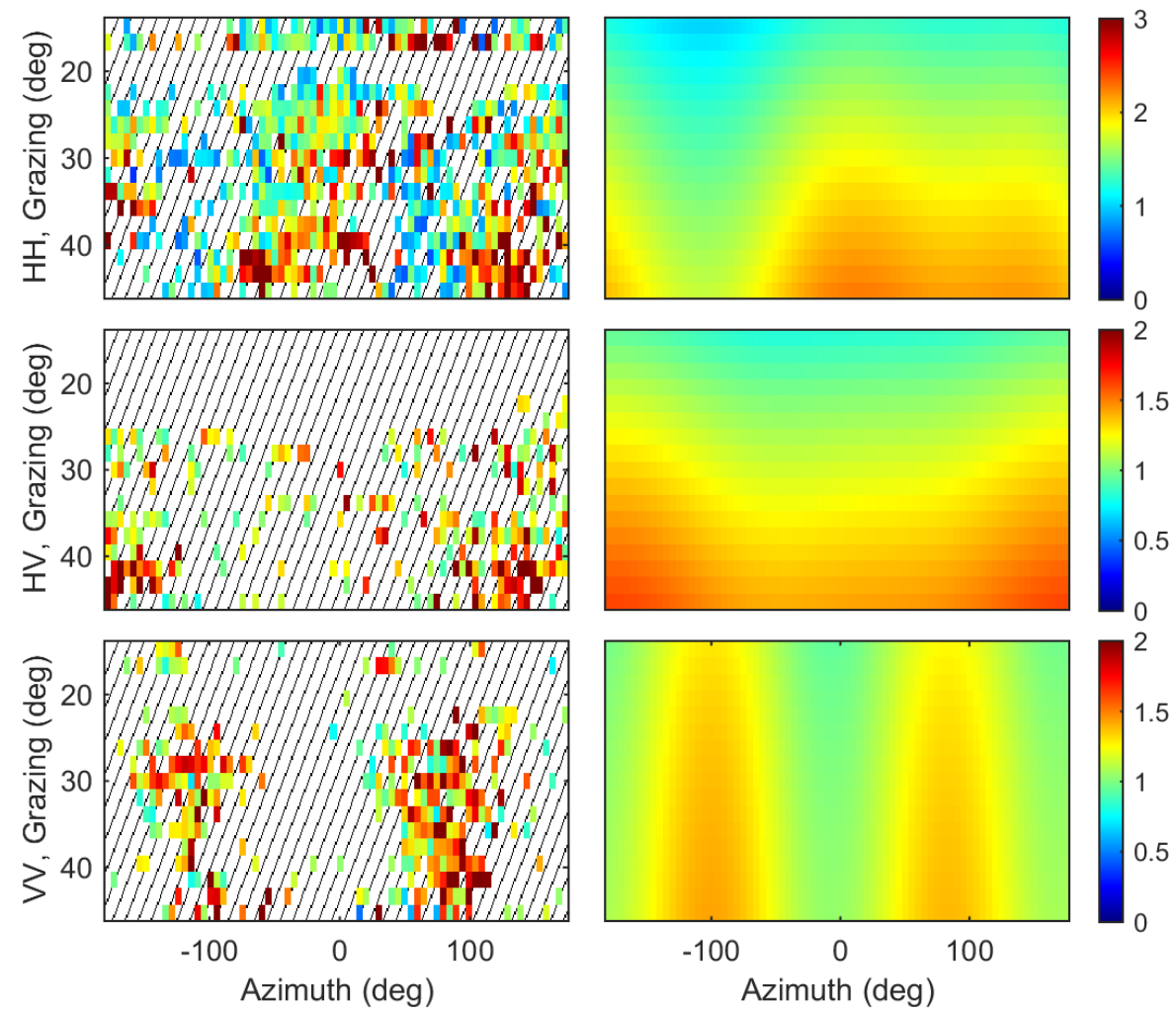

Figure 4 Variation of parameter $t$ with azimuth and grazing. Data on left, parameter model on right.
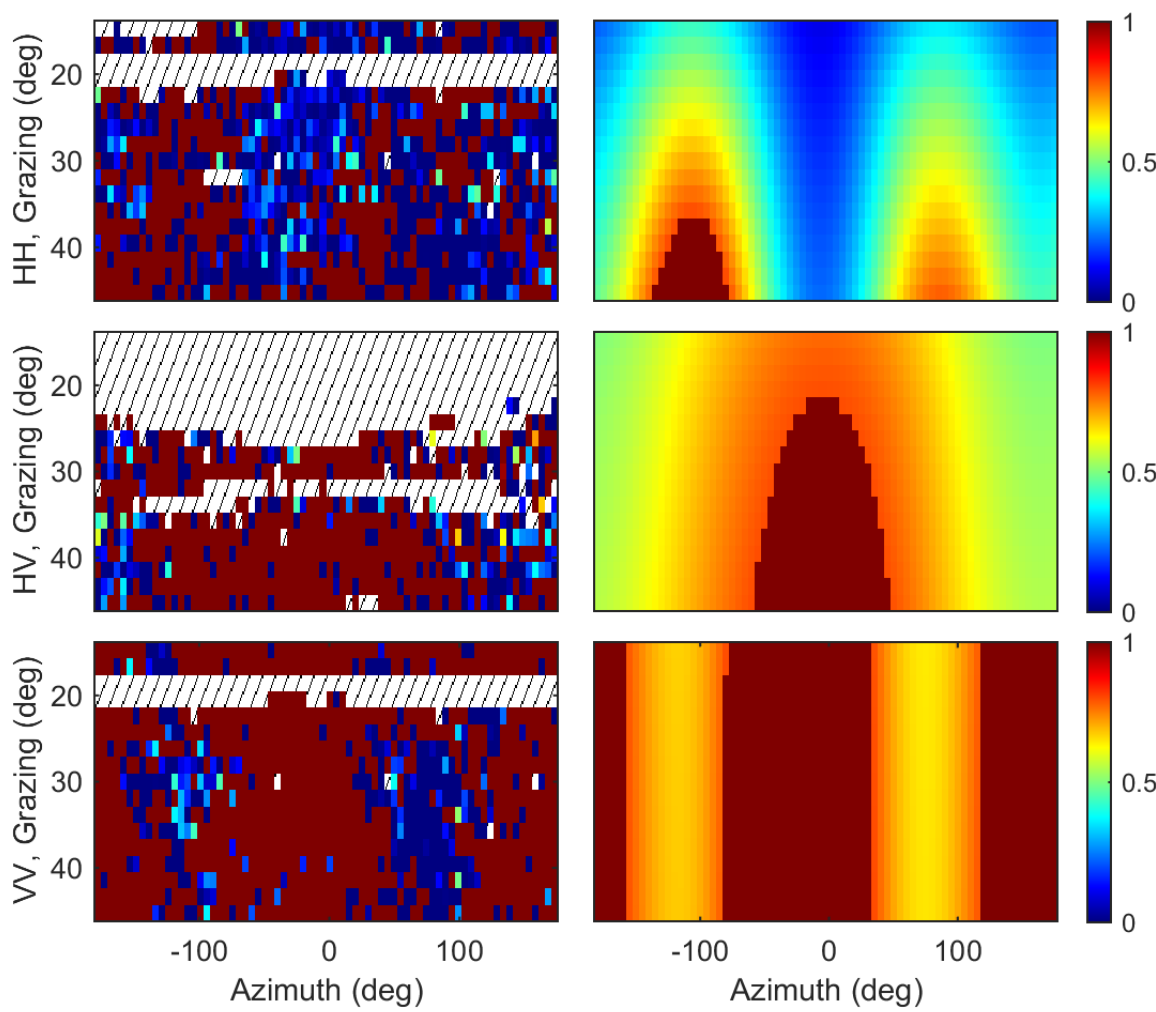

Figure 5 Variation of parameter $\beta$ with azimuth and grazing. Data on left, parameter model on right. 
Table 1 Modelled spectrum parameters

\begin{tabular}{|c|c|c|c|c|c|c|c|c|c|c|c|}
\hline \multirow{2}{*}{ Direction } & \multirow{2}{*}{ Pol. } & \multicolumn{4}{|c|}{$m_{f}(x)$} & \multirow{2}{*}{$\sigma_{r}(\mathbf{H z})$} & \multirow{2}{*}{$m_{s}(\mathbf{H z})$} & \multirow{2}{*}{$\sigma_{s}(\mathbf{H z})$} & \multirow{2}{*}{ CNR (dB) } & \multirow{2}{*}{$v$} & \multirow{2}{*}{$R(\mathbf{m})$} \\
\hline & & $A(\mathbf{H z})$ & $B(\mathrm{~Hz})$ & $t$ & $\beta$ & & & & & & \\
\hline \multirow{3}{*}{ Up-wind } & $\mathrm{HH}$ & -25.63 & 41.44 & 1.84 & 0.12 & 25.30 & 53.60 & 14.80 & 22.58 & 2.62 & 3.16 \\
\hline & $\mathrm{HV}$ & -24.40 & 56.29 & - & 1.0 & 22.19 & 55.40 & 11.44 & 11.30 & 7.18 & 1.98 \\
\hline & VV & -6.18 & 10.54 & - & 1.0 & 17.87 & 50.93 & 12.55 & 28.90 & 31.78 & 3.48 \\
\hline \multirow{3}{*}{ Cross-wind } & $\mathrm{HH}$ & -0.49 & 14.36 & 1.28 & 0.48 & 26.58 & 52.67 & 13.01 & 17.21 & 1.51 & 2.47 \\
\hline & $\mathrm{HV}$ & -6.09 & 14.43 & 1.09 & 0.66 & 24.08 & 50.77 & 10.12 & 8.61 & 1.36 & 3.91 \\
\hline & VV & 3.56 & -0.07 & - & 1.0 & 14.51 & 47.79 & 9.26 & 23.71 & 16.52 & 6.57 \\
\hline \multirow{3}{*}{ Down-wind } & $\mathrm{HH}$ & 91.80 & -58.23 & 1.14 & 0.38 & 19.07 & 43.59 & 13.04 & 18.65 & 3.28 & 2.90 \\
\hline & $\mathrm{HV}$ & 33.36 & -76.24 & 1.22 & 0.52 & 19.79 & 43.23 & 9.23 & 10.66 & 5.62 & 2.03 \\
\hline & VV & -0.32 & 2.00 & - & 1.0 & 12.29 & 40.99 & 8.35 & 27.77 & 36.07 & 5.83 \\
\hline
\end{tabular}

Table 2 Simulation parameters

\begin{tabular}{|l|l|}
\hline Parameter & Value \\
\hline Centre frequency & \\
\hline Bandwidth & $10 \mathrm{GHz}$ \\
\hline Pulse width & $200 \mathrm{MHz}$ \\
\hline PRF & $5 \mu \mathrm{s}$ \\
\hline Transmitter power & $700 \mathrm{~Hz}$ \\
\hline Antenna gain (one way) & $5 \mathrm{~kW}$ \\
\hline Combined losses & $27 \mathrm{~dB}$ \\
\hline Noise figure & $7 \mathrm{~dB}$ \\
\hline System temperature & $4 \mathrm{~dB}$ \\
\hline Beamwidth (two-way, 3dB - az. / el.) & $290 \mathrm{~K}$ \\
\hline Platform velocity & $1^{\circ} / 10^{\circ}$ \\
\hline Range & $100 \mathrm{~m} / \mathrm{s}$ \\
\hline Elevation / grazing angle $\left(^{\circ}\right)$ & $20 \mathrm{~km}$ \\
\hline Wind swell angle & $30^{\circ}$ \\
\hline Sea state & $0^{\circ}$ \\
\hline
\end{tabular}

\section{Assessment of models}

This model for the time or range varying power spectrum can be used to predict characteristics such as the variation of clutter shape parameter and CNR, as a function of Doppler frequency, $f$. As previously observed in [4], the PDFs of data in individual Doppler bins, observed over time or a range interval, are found to be well fitted by the K distribution family, with added thermal noise. Using the model, the first and second moments of the clutter intensity in the Doppler domain can now be expressed as a function of Doppler frequency, $F$, at the centre of a Doppler bin of width $\Delta f$ :

$$
\begin{aligned}
\left\langle x^{\prime}(F)\right\rangle & =\frac{1}{\Delta f} \int_{0}^{\infty} \int_{0}^{\infty} \int_{0}^{\infty} \int_{F-\Delta f / 2}^{F+\Delta f / 2} p_{c}(x) p_{s}(s) p_{r}(r) \mathbf{G}\left(f, x, s_{1}, s_{2}\right) d f d x d s d r \\
\left\langle x^{\prime}(F)^{2}\right\rangle & =\frac{1}{\Delta f} \int_{0}^{\infty} \int_{0}^{\infty} \int_{0}^{\infty} \int_{F-\Delta f / 2}^{F+\Delta f / 2} p_{c}(x) p_{s}(s) p_{r}(r) \mathbf{G}^{2}\left(f, x, s_{1}, s_{2}\right) d f d x d s d r
\end{aligned}
$$

$p_{c}(x)$ is the gamma PDF of the intensity in the time domain, $x$, with shape and scale parameters $v$ and $b$, associated with the compound $\mathrm{K}$ distribution:

$$
p_{c}(x)=\frac{b^{v}}{\Gamma(v)} x^{\nu-1} \exp (-b x)
$$

In general, we have set $s_{1}=s_{2}=s$ when modelling the data. Further work is required to develop the modelling of the two components of a bimodal spectrum, to determine the appropriate relative values of $s_{1}$ and $s_{2}$ and the correlation 
between them. As it has already been determined that the average spectrum shape for low intensity clutter returns $\left(x_{n}\right.$ $\leq t$ in (2)) is a good fit to a Gaussian shape, it would seem appropriate to set $s_{1}=s_{2}$, otherwise $\mathbf{G}\left(f, x, s_{1}, s_{2}\right)$ in $(1)$ will not be a good fit to a Gaussian shape when $x_{n} \leq t$ (see discussion in Section 2).

Fitting to the $\mathrm{K}$ distribution in each Doppler frequency, the shape parameter of the gamma-distributed component of the clutter as a function of Doppler frequency is then given by matching first and second intensity moments of the intensity at a given Doppler frequency [4]:

$$
v^{\prime}(F)=\left(\frac{\left\langle x^{\prime}(F)^{2}\right\rangle}{\left\langle x^{\prime}(F)\right\rangle^{2}}-1\right)^{-1}
$$

If the CNR of the time series data is $C$, then the noise power in each Doppler bin is given by:

$$
p_{n}=\frac{\langle x\rangle}{N C}=\frac{(v / b)}{N C}
$$

where $x, v$ and $b$ are time-domain parameters and the mean power of a K-distributed variate is $v / b$ [4]. The CNR in each Doppler bin for an idealised filter with pass-band $\Delta f$ and a centre frequency $F$ is then given by

$$
C(F)=\frac{1}{p_{n}} \int_{0}^{\infty} \int_{0}^{\infty} \int_{0}^{\infty} \int_{F-\Delta f / 2}^{F+\Delta f / 2} p_{c}(x) p_{s}(s) p_{r}(r) \mathbf{G}\left(f, x, s_{1}, s_{2}\right) d f d x d s d r
$$

Fig. 6 shows an example of the variation of the $\mathrm{K}$ distribution shape parameter, $v^{\prime}(F)$, with Doppler frequency, for the HH upwind data. For this plot, it is assumed that $r=0$. The results in Fig. 6 show clearly the effects of the rangevarying (non-stationary) Doppler spectra. The variations in mean Doppler shift and the random fluctuations in spectrum width mean that, when observed over many range cells, the clutter returns in the outer Doppler bins appear very spiky, compared with those at the centre of the spectrum. The ability to reproduce this behaviour was the driving force behind the development of this model [4]. Fig. 7 compares the actual $C(F)$ with that predicted by the model. When dealing with sampled data that has been processed with an FFT, it is possible that the spectrum will be aliased. This can be easily modelled by restricting the value of $F$ in (9) and (13) to $\pm f_{r} / 2$ and modelling $(2 m+1)$ aliased components of the spectrum as $\sum_{n=-m}^{m} \mathbf{G}\left(f+n f_{r}, x, s_{1}, s_{2}\right)$. Aliasing affects the results in Fig. 7, as indicated on the plot.

The modelling method is not restricted to representing the clutter with a $\mathrm{K}$ distribution PDF of intensity in the time domain. In (10), $p_{c}(x)$ could be any equivalent component of a compound Gaussian model, such as an inverse gamma distribution (for the Pareto model) or an offset gamma distribution (for the K-plus-Rayleigh model). Fig. 6 also shows the simulation results achieved by representing the clutter with a K-plus-Rayleigh distribution [18]. It can be seen in both examples that the model reproduces the salient characteristics of the data quite well. 
Correctly modelling any bimodal characteristics of the clutter will affect how accurately the behaviour at the edges of the spectrum are represented. From inspection of (2), it can be seen that, if $\alpha \neq 0$, applying a linear fit to the clutter mean Doppler, $m_{f}(x)$, would predict values that are either too low or high for intensity values above the threshold, $t$. The predicted values of $v^{\prime}(F)$ at the edge of the spectrum will be too low if the bimodal behaviour is ignored, although in many cases this effect will be quite small. The spiky behaviour at the edges of the spectrum is caused by the fluctuations in the spectrum width and the change in mean Doppler shift. However, in this case the change in predicted mean Doppler shift between the bimodal model and linear model (i.e. setting $\beta=1$ ) has a relatively small effect compared with the fluctuations in spectrum width. The effects of modelling the additional fluctuations due to $r$ are also shown in Fig. 8. Including this parameter has the effect of predicting spikier behaviour in the edges of the spectrum, as well as in the centre of the spectrum. When compared with the shape parameter of the real data near the centre of the spectrum, the predicted effect of $r$ appears rather strong. This may be because the parameters $s, x$ and $r$ are treated as independent variables in (9) and it is possible that these components are in fact partially correlated. Also, direct comparisons with the original data are a guide to performance of the models but it must be remembered that the original data sets were not very large (about 1800 spectra in each group of 200 range cells by 9 pulse bursts). This means that estimates of shape parameters from the original data may differ from predictions obtained by averaging over all possible parameter values in the model. The simulations discussed in the Section 5 will equally show variation from batch to batch if comparably sized data sets are generated.

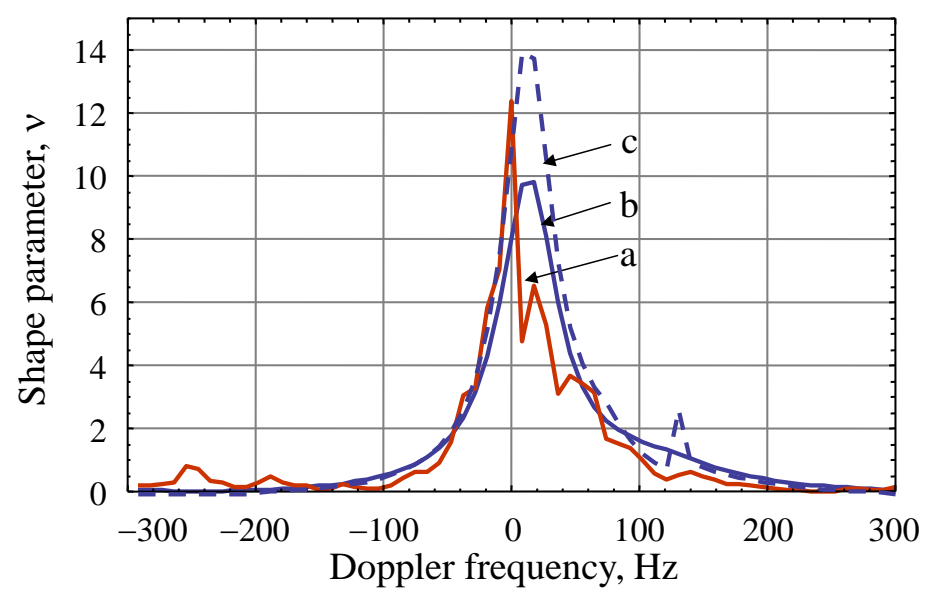

Figure $6 \mathrm{~K}$ distribution shape parameter $v^{\prime}(f)$ for clutter component alone as a function of Doppler frequency (HH pol, upwind).

a: HH pol data;

b: model prediction assuming data has a $\mathrm{K}$ distribution in the time domain $(v=4.3)$ and with $A=29 \mathrm{~Hz}, B=28$

$\mathrm{Hz}, t=2.5, \beta=0.5, m_{s}=55 \mathrm{~Hz}, \sigma_{s}=19 \mathrm{~Hz}$;

c: model prediction assuming data has a K-plus-Rayleigh distribution in the time domain. 


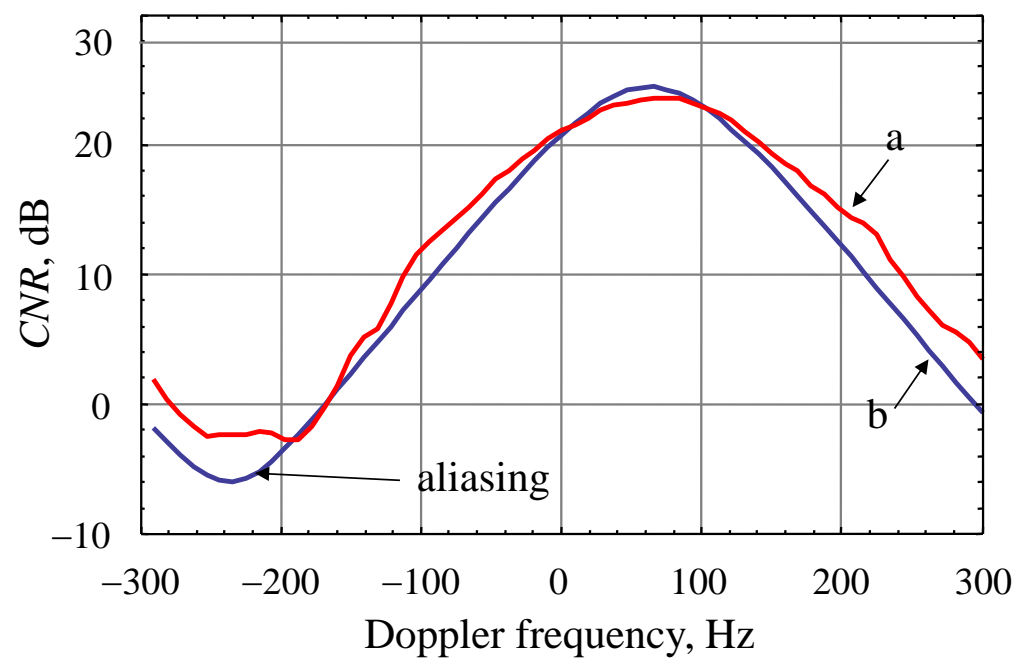

Figure 7 CNR as a function of Doppler frequency. a: HH pol data; b: model prediction.

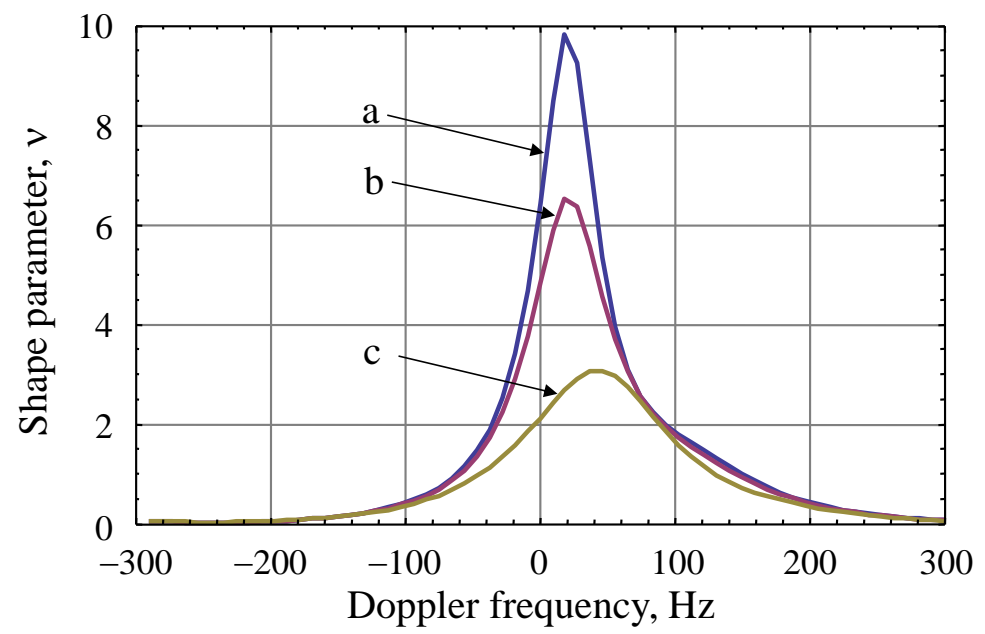

Figure 8 Modelled variation of shape parameter as a function of Doppler frequency for a: Bi-modal model prediction, $r=0$ (see Fig. 6);

b: Bi-modal model prediction, $\sigma_{r}=10 \mathrm{~Hz}$;

c: Bi-modal model prediction, $\sigma_{r}=25 \mathrm{~Hz}$.

\section{Simulation}

An additional application for this modelling method is the simulation of coherent Doppler spectra and time domain data [4]. In addition to the parameters described above, the range correlation coefficients of the spectrum intensity, $x$, and the spectrum standard deviation, $s$, are required. Fig. 9 shows the correlation coefficients of intensity, $x$, and spectrum standard deviation, $s$, for data over range cells $1-200$. The correlation coefficient of the spectrum centroid is very similar to that of the intensity. The data was simulated by generating correlated gamma samples with appropriate correlation in range. The intensity has a shape parameter $v=2.23$ and the standard deviation, $s$, had shape and scale parameters derived from $m_{s}=55.5 \mathrm{~Hz}$ and $\sigma_{s}=19.6 \mathrm{~Hz}$, as shown in (4). For each position in range, a complex spectrum is generated using the square root of a sampled version of the power spectrum given in (1), with $s_{1}=s_{2}=s, A=-59.4$ $\mathrm{Hz}, B=58.06 \mathrm{~Hz}, t=2.21$ and $\beta=0$ (see Table 2 in [1]). The centre frequency of each spectrum is obtained from $m_{f}\left(x_{n}\right)$ 
combined with a sample from a correlated Gaussian sequence with zero mean and a standard deviation of $\sigma_{r}=30.47 \mathrm{~Hz}$. The correlation coefficient of the intensity is also used for the centre frequency. Setting $s_{1}=s_{2}$ has the effect of giving the two spectrum components the same standard deviation, consistent with the results of the data analysis. This voltage spectrum modulates random zero-mean complex Gaussian samples in each Doppler bin. The bi-modal spectra were then convolved with the azimuth beam pattern of the Ingara radar and uncorrelated complex normal variates were added to simulate thermal noise at the appropriate CNR. Fig. 10 compares measured and modelled power spectra over a range interval of 200 range samples for $\mathrm{HH}$ upwind data. The results are qualitatively similar and reproduce the same statistics in the Doppler domain, as shown in Fig. 11.

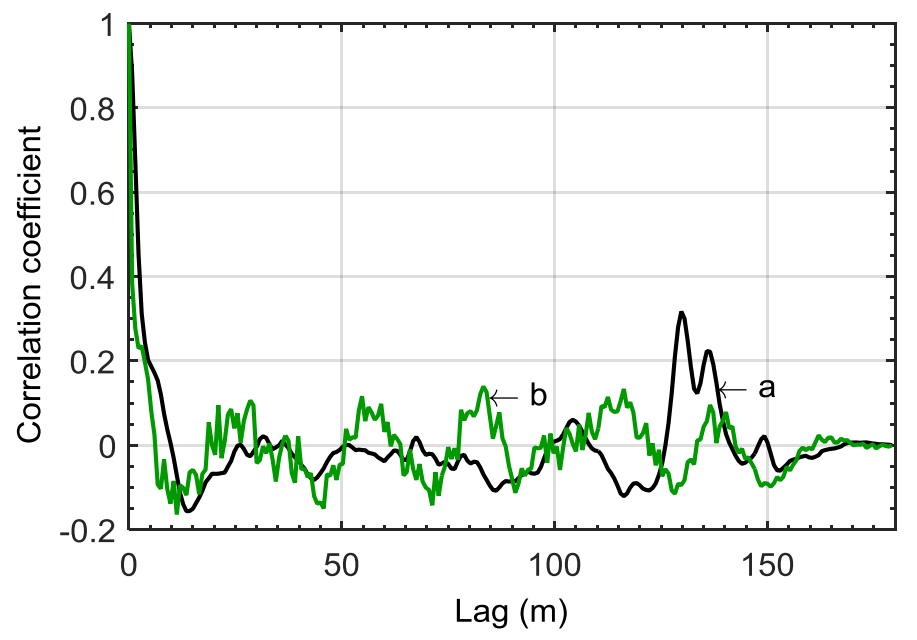

Figure 9 Correlation coefficient of (a) clutter intensity, $x$, and (b) spectrum standard deviation, $s$, over range (HH Pol., up-wind, range cells 1 -200).
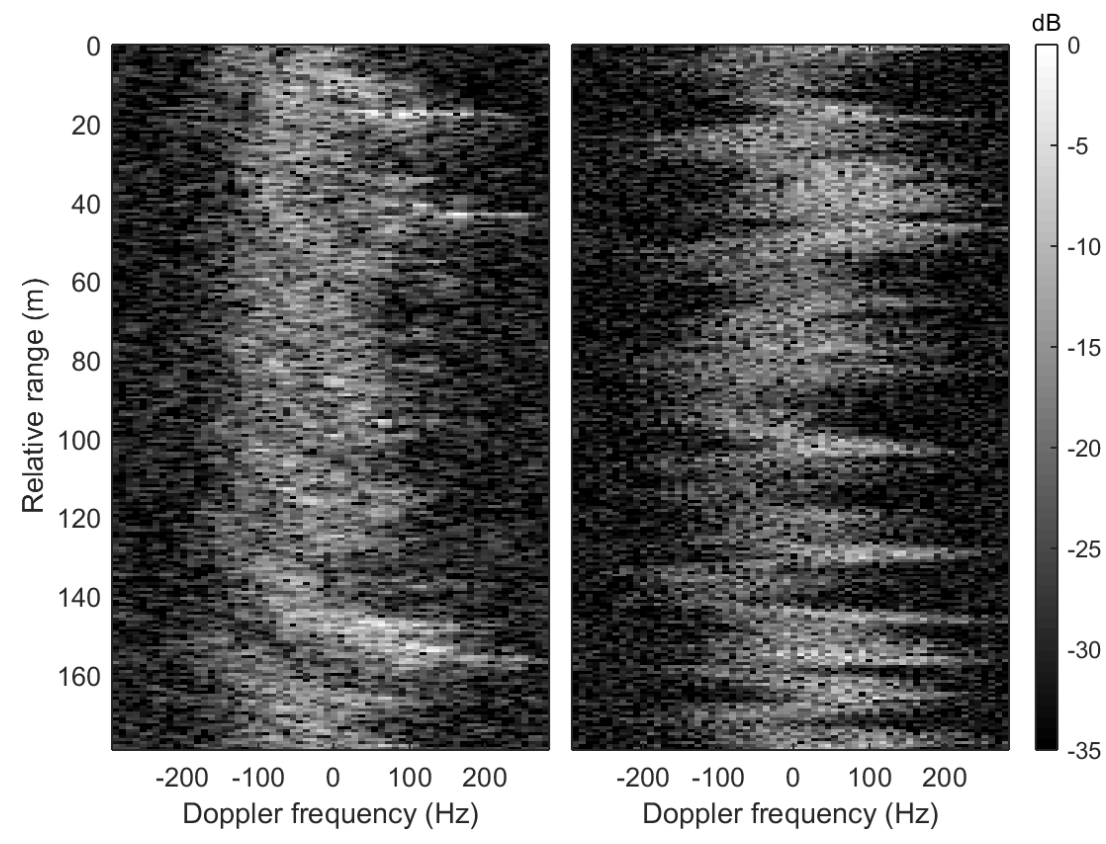

Figure 10 Power spectra: left, HH pol up-wind, range cells 1 - 200; right: simulated data with model parameters taken from same data. 
In order to characterise the model as a means of generating clutter spikes, a sequence of $10^{6}$ correlated gamma variates was generated, using the parameters from the upwind $\mathrm{HH}$ polarisation case in Fig. 10 and the correlation coefficient shown in Fig. 9(a) (the correlation coefficient is set to zero for lags greater than $150 \mathrm{~m}$.). Variates exceeding the bimodal model threshold $t=2.21$ are considered spikes. The Doppler spectrum of the spikes is narrower than the overall mean Doppler spectrum (Fig. 11), with a higher centre frequency. The range extent of the spikes is measured as the number of consecutive variates above the threshold in the correlated gamma sequence. A large number of spikes appear in only one range bin, but there are also numerous spikes extending over two or more bins. On a log scale the number of spikes decreases approximately linearly with increasing length, consistent with a power law relationship (Fig. 12).

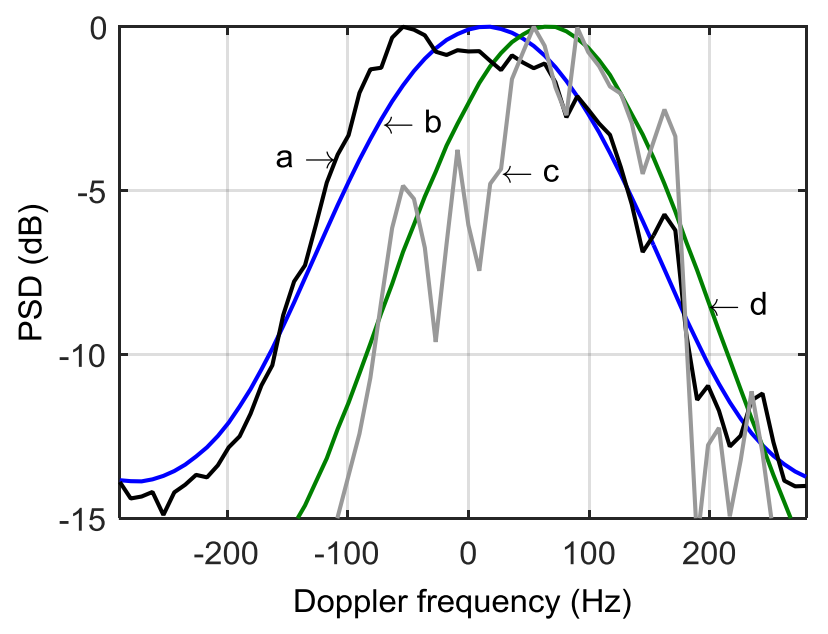

Figure 11 Mean Doppler spectrum from data (a) and simulation (b) with spike Doppler spectrum from data(c) and simulation (d).

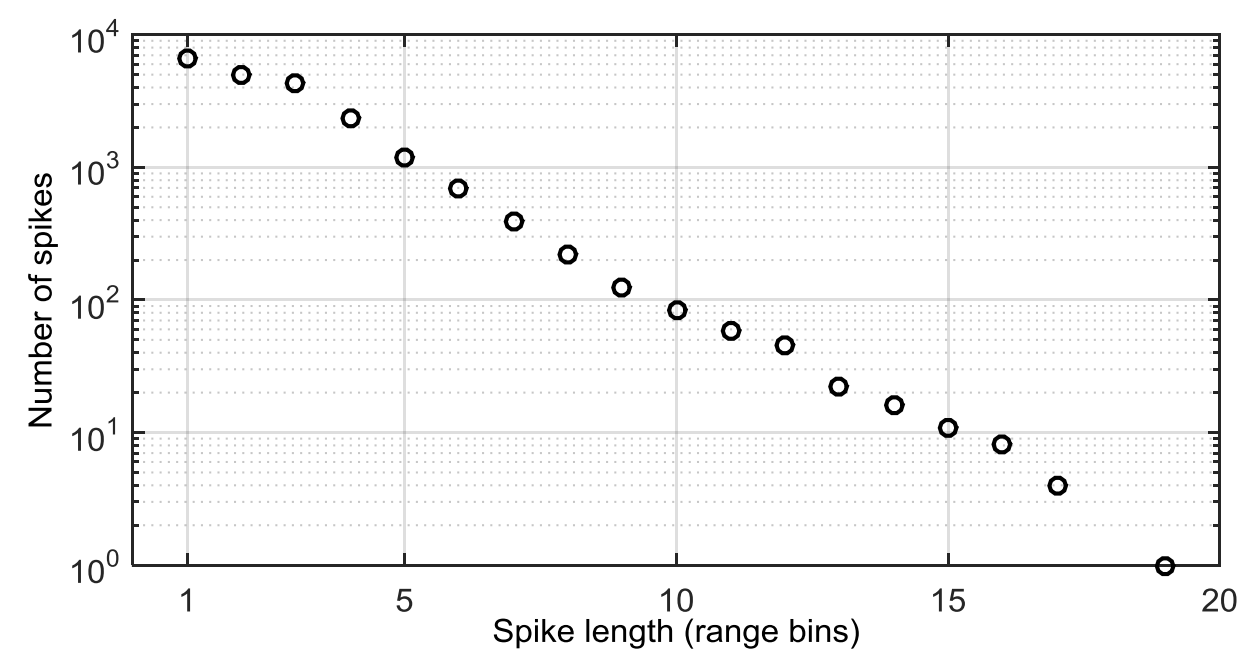

Figure 12 Number of spikes vs spike extent in range. 
To demonstrate the parameter models described in Section 3.1, a set of simulations based on the example parameters in Tables 1 and 2 was produced (Fig. 13). Each of the nine examples in Fig. 13 was constructed from three sequences of correlated random variates, one each for the spectrum intensity, width and centroid fluctuations about the mean. A Gaussian model was used for the correlation coefficient, $A$, over range $y$ of each sequence, with the correlation length $R$ from Table 1:

$$
A(y)=\exp \left(-\frac{y^{2}}{R^{2}}\right)
$$

The same random seed was used to construct the correlated sequences for the spectrum intensity in the different polarisations, as the spectra arise from the same underlying sea surface structure. Different random seeds were used for the different look directions. The spectrum intensity is gamma distributed with unit mean and shape parameter $v$. The spectrum width is also gamma distributed, with the shape and scale derived from the mean $m_{s}$ and standard deviation $\sigma_{s}$ in Table 1 , following (4). The centroid fluctuations are normally distributed with standard deviation $\sigma_{r}$. Thermal noise is represented by adding uncorrelated complex normal variates to produce the required CNR. The simulations reproduce the typical characteristics of clutter spectra. The HH polarisation spectra show the most spikes and the VV polarisation spectra the least. The CNR is noticeably lower in HV polarisation than in the other polarisations. In the upwind spectra the spikes are predominantly at positive Doppler frequencies whereas in the downwind spectra they occur at negative Doppler frequencies. In the crosswind case the Doppler shift associated with the spikes is smaller, with positive and negative displacements equally likely. 


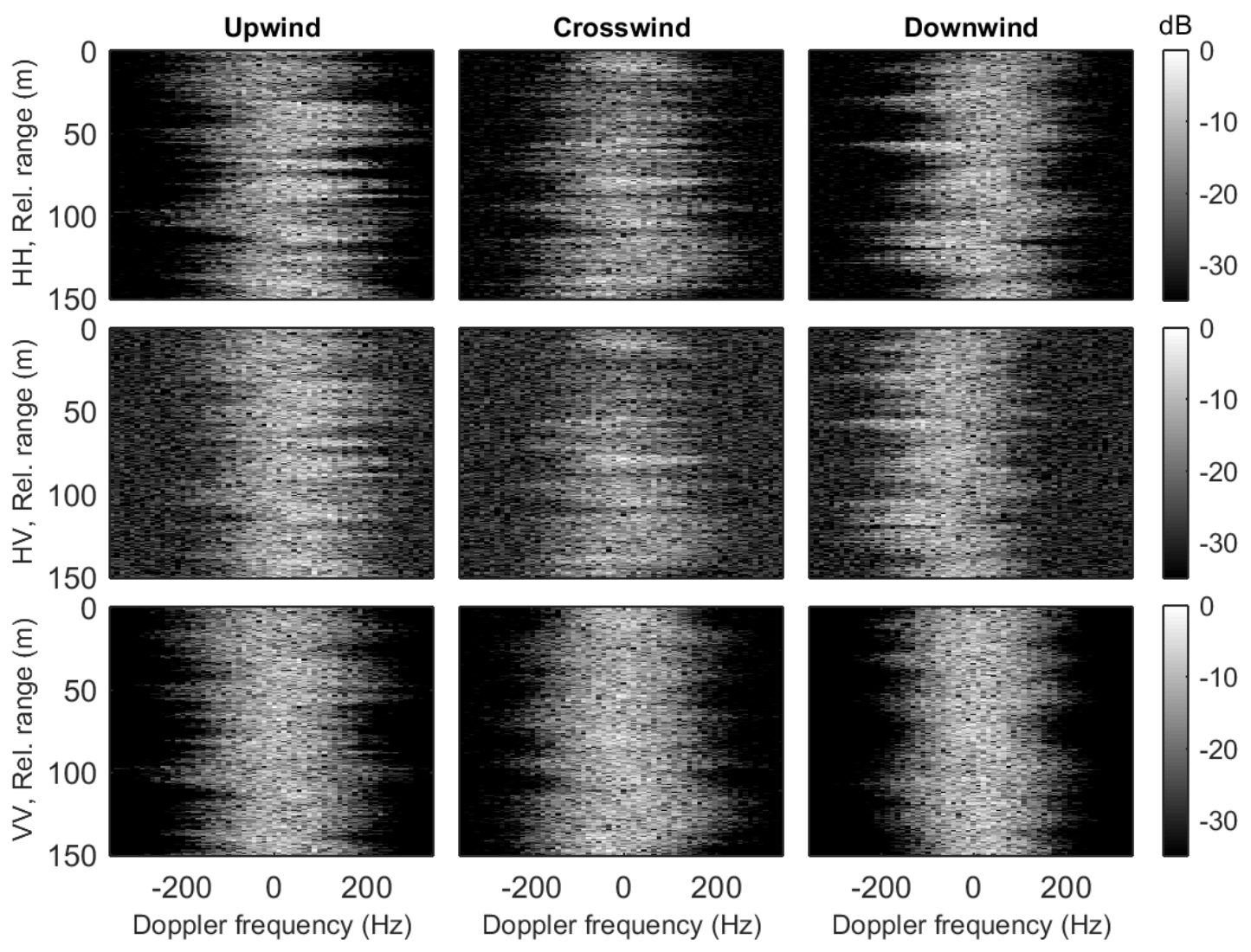

Figure 13 Examples of simulated data

\section{Comparisons with low grazing angle results}

The original derivation of these models was based on analysis of the CSIR Fynmeet database [19], with X-band data collected at grazing angles of about $0.1^{\circ}$. It has been shown here that the basic modelling method is equally applicable to data at both low and medium grazing angles. In both cases a strong variation in the linear relationship of the PSD centre frequency with intensity was found as a function of bearing with respect to wind and wave direction. Relative increases in the shape parameter of the K distribution PDF, with added thermal noise, were also shown in the medium grazing angle Ingara data. In addition, analysis showed that the location of the peaks in the $\mathrm{K}$ distribution shape parameter, with respect to wind direction, were at comparable locations in both low and medium grazing data. Comparisons of the Ingara and CSIR data and their Doppler spectra were reported in [20]. One of the characteristics of the medium grazing angle Ingara data that was observed was an excellent fit of the intensity to a K-plus-Rayleigh compound distribution [18]. This model is very similar to a compound $\mathrm{K}$ distribution with added thermal noise, but it is found that in many cases the Rayleigh amplitude component is much greater than that which would be due to the known thermal noise level. The CSIR data has been assessed to see if it is also a good fit to this family of distributions. This was completed using moment analysis of the data to estimate the thermal noise component and comparing this to the actual thermal noise power. Significant increases in the estimated thermal noise component would indicate a 
strong additional Rayleigh component. However, from this analysis it was found that in fact a standard compound Kdistribution, with added thermal noise as appropriate, was a much better fit to that data.

\section{Conclusions}

This paper, together with Part 1 [1], has shown that the Doppler spectra of radar sea clutter data collected at multiple polarisations, multiple look directions and at medium grazing angles can be well represented by the modelling method originally reported in [4]. This paper has shown how the essential characteristics of the Doppler spectra, including their correlations over range and time, can be represented realistically in a model. It is further shown that these models can be used in analytic modelling of radar performance and in the simulation of realistic coherent clutter returns.

As a refinement of the model, it was observed that in some conditions a further broadening of spectra was observed when the local clutter intensity exceeded some threshold level, resulting in apparently bimodal spectra. This bimodal behaviour has also been captured in the model and the paper describes how these bimodal characteristics vary with clutter conditions and radar polarisation.

To be of value to a radar designer, models of clutter must include any dependencies on radar parameters and environmental conditions. The methodology for capturing this variation has been described in the paper and applied to the parameters used to model bimodal behaviour.

\section{Acknowledgement}

This work was undertaken as part of the NATO SET-185 Task Group. We also acknowledge both the DST Group and CSIR for providing the Ingara and Fynmeet data sets.

\section{References}

[1] Watts, S., Rosenberg, L., Ritchie, M. and Bocquet, S., The Doppler spectra of medium grazing angle sea clutter; Part 1: Characterisation, IET RSN (part 1 of this paper series).

[2] Crisp, D.J., Stacy, N.J., and Goh, A.S., "Ingara medium-high incidence angle polarimetric sea clutter measurements and analysis,” DSTO, Technical Report DSTO-TR-1818, February 2006.

[3] Watts, S., "A new method for the simulation of coherent sea clutter," IEEE Radar Conference, pp. 52-57, May 2011.

[4] Watts, S., Modeling and simulation of coherent sea clutter, IEEE Trans AES Vol. 48, No.4, October 2012, pp. 3303-3317.

[5] Watts, S., Rosenberg, L., and Ritchie, M., "Characterising the Doppler spectra of high grazing angle sea clutter," in IEEE International Radar Conference, October 2014. 
[6] Rosenberg, L., Watts, S., Bocquet, S. and Ritchie, M. "Characterisation of the Ingara HGA dataset", IEEE International Radar Conference, May 2015

[7] M. Greco, F. Bordoni, F. Gini, "X-band sea clutter non-stationarity: the influence of long waves,” IEEE Journal on Ocean Engineering, Special Issue on "Non-Rayleigh Reverberation and Clutter", special issue, Vol.29, No. 2, April 2004, pp.269-283.

[8] Lee P.H.Y., Barter J.D. Beach K.L., Caponi, E., Hindman C.L., Lake B.M., Rungaldier H., and Shelton J.C.: 'Power spectral lineshapes of microwave radiation backscattered from sea surfaces at small grazing angles', lEE Proc. F, October 1995, 142, (5), pp. 252-258.

[9] Rozenberg A.D., Quigley D.C., Melville W.K., "Laboratory study of polarized microwave scattering by surface waves at grazing incidence: the influence of long waves," IEEE Trans. on Geoscience and Remote Sensing, Vol.34, No. 6, November 1996, pp. 1331-1342.

[10] Trizna D.B., "A model for Doppler peak spectral shift for low grazing angle sea scatter," IEEE Journal of Oceanic Engineering, Vol. 10, No. 4, October 1985, pp. 368-375.

[11] Davidson, G., Simulation of coherent sea clutter, IET Radar Sonar Navig.,Vol. 4, Iss. 2, April 2010, pp. 168 177 (Special Issue on Radar Clutter).

[12] Miller, R. J., Variability in spectra of low-grazing angle sea clutter returns, NATO/RTO Publications, Proceedings of SET Symposium on Low Grazing Angle Clutter: Its Characterisation, Measurement and Application, April 2000 .

[13] Bocquet, S., Rosenberg, L., Watts, S., 'Simulation of coherent sea clutter with inverse gamma texture', International Conference Radar 2014, October 2014.

[14] Crisp, D.J., Rosenberg, L., and Stacy, N.J., "Modelling ocean backscatter in the plateau region at X-band with the K-distribution”, DSTO Technical Report, 2015.

[15] Crisp, D.J., Kyprianou, R., Rosenberg, L., and Stacy N.J., "Modelling X-band sea clutter at moderate grazing angles”, IEEE International Radar Conference, September 2008, pp. 596-601.

[16] Rosenberg, L., and Watts, S. "High grazing angle sea-clutter literature review”, General Document, DSTO-GD0736, 2013.

[17] Technology Service Corporation, Backscatter from sea, Radar Workstation, 1990, vol. 2, 177-186.

[18] Rosenberg, L., Watts, S., Bocquet, S., 'Application of the K+Rayleigh distribution to high grazing angle seaclutter', International Conference Radar 2014, October 2014.

[19] CSIR, small boat detection research, http://www.csir.co.za/small_boat_detection/datasets.html. 
[20] Ritchie, M.A., Watts, S., Rosenberg, L., Griffiths, H.D., Statistical comparison of low and high grazing angle sea clutter, International Conference Radar 2014, October 2014. 


\section{Appendix}

The sets of model coefficients for the parameters described in Section 3.1 are listed in Tables A1-A4. The coefficients for the $\mathrm{K}$ and Pareto shape parameters were given in [6].

Table A 1 Bi-modal parameter model coefficients for $A$ and $B$.

\begin{tabular}{|c|c|c|c|c|c|c|c|}
\hline \multirow{2}{*}{ Polarisation } & \multirow{2}{*}{ Coeff. } & \multicolumn{3}{|c|}{$A$} & \multicolumn{3}{|c|}{$B$} \\
\hline & & $b_{0}$ & $b_{1}$ & $b_{2}$ & $b_{0}$ & $b_{1}$ & $b_{2}$ \\
\hline \multirow{6}{*}{$\mathrm{HH}$} & $\gamma$ & -2.38 & 2.77 & 0.044 & -1.88 & 1.44 & 0.059 \\
\hline & $\alpha_{0}$ & 127.9 & -125.1 & -0.46 & -1.98 & 5.62 & -0.018 \\
\hline & $\alpha_{1}$ & -262.3 & 225.7 & 3.43 & 148.9 & -103.6 & -5.03 \\
\hline & $\alpha_{2}$ & 126.1 & -123.6 & -1.29 & -85.09 & 81.82 & 0.58 \\
\hline & $\alpha_{3}$ & 6.81 & -15.26 & 3.69 & -28.09 & 38.42 & -4.21 \\
\hline & $\alpha_{4}$ & 6.71 & -4.54 & -0.38 & 4.50 & -4.30 & -0.26 \\
\hline \multirow{6}{*}{ HV } & $\gamma$ & 5.85 & -3.73 & -0.54 & 2.65 & -2.95 & -0.094 \\
\hline & $\alpha_{0}$ & -18.1 & 19.57 & -0.045 & 5.03 & -2.05 & -0.69 \\
\hline & $\alpha_{1}$ & -40.31 & 24.39 & -2.56 & 147.3 & -101.3 & -1.34 \\
\hline & $\alpha_{2}$ & 18.09 & -16.47 & -0.23 & -47.33 & 45.75 & -0.92 \\
\hline & $\alpha_{3}$ & -37.14 & 35.17 & -0.43 & 49.71 & -42.13 & -1.14 \\
\hline & $\alpha_{4}$ & 11.2 & -10.07 & -0.094 & -21.95 & 21.32 & -0.76 \\
\hline \multirow{6}{*}{ VV } & $\gamma$ & -3.01 & 3.86 & -0.23 & -2.79 & 3.22 & -0.10 \\
\hline & $\alpha_{0}$ & 0.24 & -0.14 & 0.028 & 7.45 & -6.87 & 1.22 \\
\hline & $\alpha_{1}$ & -8.63 & -13.65 & 9.85 & 16.37 & 6.29 & -9.86 \\
\hline & $\alpha_{2}$ & -4.33 & 5.26 & -2.18 & 1.29 & -2.37 & 2.23 \\
\hline & $\alpha_{3}$ & 0.72 & -2.75 & 3.85 & -2.90 & 5.56 & -3.97 \\
\hline & $\alpha_{4}$ & -2.08 & 3.93 & -1.43 & 0.13 & -1.15 & 1.19 \\
\hline
\end{tabular}

Table A 2 Bi-modal parameter model coefficients, for $t$ and $\beta$.

\begin{tabular}{|c|c|c|c|c|c|c|c|}
\hline \multirow{2}{*}{ Polarisation } & \multirow{2}{*}{ Coeff. } & \multicolumn{3}{|c|}{$\mathbf{t}$} & \multicolumn{3}{|c|}{$\beta$} \\
\hline & & $b_{0}$ & $b_{1}$ & $b_{2}$ & $b_{0}$ & $b_{1}$ & $b_{2}$ \\
\hline \multirow{6}{*}{$\mathrm{HH}$} & $\gamma$ & 5.27 & -4.48 & -0.11 & 7.41 & -5.96 & -0.28 \\
\hline & $\alpha_{0}$ & -0.55 & 2.15 & 0.027 & -0.21 & 0.65 & 0.0025 \\
\hline & $\alpha_{1}$ & -0.24 & 0.40 & 0.035 & 0.15 & -0.25 & -0.0098 \\
\hline & $\alpha_{2}$ & 0.42 & -0.45 & 0.045 & 0.25 & -0.37 & -0.024 \\
\hline & $\alpha_{3}$ & -0.21 & 0.45 & 0 & 0.069 & -0.12 & -0.0028 \\
\hline & $\alpha_{4}$ & -0.024 & 0.0016 & 0.024 & -0.034 & 0.13 & -0.066 \\
\hline \multirow{6}{*}{ HV } & $\gamma$ & 0.41 & 0.25 & -0.084 & -1.96 & 2.03 & -0.0048 \\
\hline & $\alpha_{0}$ & 0.59 & 0.56 & 0.030 & 0.62 & 0.11 & -0.018 \\
\hline & $\alpha_{1}$ & 0.096 & -0.22 & 0.020 & 0.019 & 0.15 & -0.021 \\
\hline & $\alpha_{2}$ & 0.026 & 0.11 & -0.045 & 0.48 & -0.54 & 0.026 \\
\hline & $\alpha_{3}$ & 0.017 & -0.031 & 0.012 & 0.76 & -0.72 & -0.026 \\
\hline & $\alpha_{4}$ & -0.31 & 0.35 & -0.019 & 0.095 & -0.11 & 0.0021 \\
\hline \multirow{6}{*}{ VV } & $\gamma$ & 0.031 & 0.22 & -0.065 & 0.097 & -0.080 & -0.0039 \\
\hline & $\alpha_{0}$ & 1.00 & 0.11 & 0.031 & 0.89 & 0.18 & -0.090 \\
\hline & $\alpha_{1}$ & 0.10 & -0.11 & -0.0095 & 0.021 & -0.06 & 0.025 \\
\hline & $\alpha_{2}$ & 0.23 & -0.31 & -0.030 & -0.043 & 0.016 & 0.059 \\
\hline & $\alpha_{3}$ & 0.25 & -0.40 & 0.053 & 0.059 & -0.089 & -0.0090 \\
\hline & $\alpha_{4}$ & 0.13 & -0.16 & -0.0087 & -0.038 & -0.0058 & 0.061 \\
\hline
\end{tabular}

Table A 3 Parameter model coefficients for the mean Doppler spread, $\sigma_{r}$ and spatial decorrelation length, $R$.

\begin{tabular}{|c|c|c|c|c|c|c|c|}
\hline \multirow{2}{*}{ Polarisation } & \multirow{2}{*}{ Coeff. } & \multicolumn{3}{|c|}{$\boldsymbol{\sigma}_{\boldsymbol{r}}$} & \multicolumn{3}{|c|}{$\boldsymbol{R}$} \\
\cline { 2 - 8 } & & $\boldsymbol{b}_{\mathbf{0}}$ & $\boldsymbol{b}_{\mathbf{1}}$ & $\boldsymbol{b}_{\mathbf{2}}$ & $\boldsymbol{b}_{\mathbf{0}}$ & $\boldsymbol{b}_{\mathbf{1}}$ & $\boldsymbol{b}_{\mathbf{2}}$ \\
\hline \multirow{5}{*}{$\mathrm{HH}$} & $\gamma$ & -1.07 & 0.27 & 0.12 & 1.24 & -1.32 & 0.11 \\
\cline { 2 - 8 } & $\alpha_{0}$ & 38.31 & -16.22 & 1.42 & -1.14 & 3.62 & 0.47 \\
\cline { 2 - 8 } & $\alpha_{1}$ & -10.37 & 9.67 & 0.63 & 0.28 & -0.098 & 0.093 \\
\cline { 2 - 8 } & $\alpha_{2}$ & 9.76 & -12.71 & 0.27 & -0.67 & 0.89 & 0.20 \\
\cline { 2 - 8 } & $\alpha_{3}$ & -12.38 & 13.83 & 0.92 & 0.33 & -0.50 & -0.054 \\
\cline { 2 - 8 } & $\alpha_{4}$ & 5.48 & -4.54 & -0.33 & 0.30 & -0.88 & 0.26 \\
\hline \multirow{5}{*}{$\mathrm{HV}$} & $\gamma$ & -0.0016 & -0.39 & 0.055 & -1.65 & 1.66 & -0.11 \\
\cline { 2 - 8 } & $\alpha_{0}$ & 25.65 & -5.35 & 1.31 & -3.89 & 6.59 & 0.70 \\
\cline { 2 - 8 } & $\alpha_{1}$ & 4.83 & -5.94 & 0.60 & 2.99 & -3.61 & 0.62 \\
\cline { 2 - 8 } & $\alpha_{2}$ & 0.72 & -3.57 & 0.24 & -3.58 & 3.67 & 0.12 \\
\cline { 2 - 8 } & $\alpha_{3}$ & -6.00 & 4.90 & 1.46 & -3.10 & 2.58 & 0.082 \\
\cline { 2 - 8 } & $\alpha_{4}$ & 15.33 & -14.05 & -0.32 & -7.44 & 8.23 & -0.45 \\
\cline { 2 - 8 } & $\gamma$ & -0.22 & 0.23 & 0.0043 & 0.45 & -0.57 & -0.12 \\
\cline { 2 - 8 } & $\alpha_{0}$ & 10.14 & 1.28 & 2.50 & 2.52 & 0.66 & 1.76 \\
\cline { 2 - 8 } & $\alpha_{1}$ & 1.56 & -1.34 & 0.58 & 1.55 & -1.88 & -0.34 \\
\cline { 2 - 8 } & $\alpha_{2}$ & -0.94 & 1.63 & 0.22 & 1.07 & -1.92 & 0.011 \\
\cline { 2 - 8 } & $\alpha_{3}$ & -1.77 & 2.98 & 0.51 & -0.30 & -0.32 & 0.0043 \\
\cline { 2 - 7 } & $\alpha_{4}$ & 1.79 & -3.73 & 0.76 & -0.70 & 1.78 & -0.86 \\
\hline
\end{tabular}


Table A 4 Parameter model coefficients, for $m_{s}$ and $\sigma_{s}$

\begin{tabular}{|c|c|c|c|c|c|c|c|}
\hline \multirow{2}{*}{ Polarisation } & \multirow{2}{*}{ Coeff. } & \multicolumn{3}{|c|}{$m_{s}$} & \multicolumn{3}{|c|}{$\sigma_{s}$} \\
\hline & & $b_{0}$ & $b_{1}$ & $b_{2}$ & $b_{0}$ & $b_{1}$ & $b_{2}$ \\
\hline \multirow{6}{*}{$\mathrm{HH}$} & $\gamma$ & 0.49 & -0.79 & 0.013 & 0.95 & -1.27 & 0.056 \\
\hline & $\alpha_{0}$ & 22.06 & 31.27 & 0.59 & 14.24 & -2.23 & 0.84 \\
\hline & $\alpha_{1}$ & 11.57 & -12.50 & 1.30 & -13.31 & 13.43 & 0.60 \\
\hline & $\alpha_{2}$ & -5.29 & 3.80 & 0.20 & 4.71 & -4.60 & 0.026 \\
\hline & $\alpha_{3}$ & 3.74 & -3.73 & 1.56 & -2.21 & 3.24 & 0.54 \\
\hline & $\alpha_{4}$ & -2.96 & 2.51 & 0.23 & 0.53 & -1.15 & 0.20 \\
\hline \multirow{6}{*}{$\mathrm{HV}$} & $\gamma$ & 1.14 & -1.15 & 0.0030 & 2.28 & -2.50 & 0.085 \\
\hline & $\alpha_{0}$ & 37.44 & 13.76 & 0.28 & 4.61 & 4.91 & 0.88 \\
\hline & $\alpha_{1}$ & 11.89 & -11.65 & 1.01 & 7.95 & -8.87 & 0.54 \\
\hline & $\alpha_{2}$ & -5.30 & 5.17 & -0.17 & -2.23 & 2.25 & 0.022 \\
\hline & $\alpha_{3}$ & -1.35 & 2.65 & 1.47 & -4.33 & 4.12 & 0.65 \\
\hline & $\alpha_{4}$ & 4.12 & -4.73 & 0.21 & 3.78 & -4.34 & 0.26 \\
\hline \multirow{6}{*}{ VV } & $\gamma$ & -0.20 & 0.16 & 0.0022 & 0.018 & 0.10 & 0.0016 \\
\hline & $\alpha_{0}$ & 46.05 & -0.16 & 0.68 & 4.91 & 3.52 & 1.28 \\
\hline & $\alpha_{1}$ & \begin{tabular}{|l|}
-4.19 \\
\end{tabular} & 6.26 & 0.61 & 0.39 & 0.071 & 0.35 \\
\hline & $\alpha_{2}$ & -1.24 & 0.62 & -0.081 & -1.03 & 1.92 & 0.13 \\
\hline & $\alpha_{3}$ & -4.04 & 6.70 & 0.59 & -1.36 & 2.33 & 0.31 \\
\hline & $\alpha_{4}$ & 0.32 & -0.26 & -0.14 & 0.21 & -1.094 & 0.34 \\
\hline
\end{tabular}

